\title{
Cognição na doença de Chagas: Uma revisão sistemática
}

\author{
Cognition in Chagas disease: A systematic review \\ Cognición en la enfermedad de Chagas: Una revisión sistemática
}

Recebido: 25/06/2021 | Revisado: 02/07/2021 | Aceito: 10/07/2021 | Publicado: 20/07/2021

\author{
Natalia Ligabô dos Santos \\ ORCID: https://orcid.org/0000-0002-0276-3904 \\ Universidade do Oeste Paulista, Brasil \\ E-mail: natalialigabo@gmail.com \\ Laysa Rodrigues dos Reis \\ ORCID: https://orcid.org/0000-0002-3673-6015 \\ Universidade do Oeste Paulista, Brasil \\ E-mail: laysarreis@gmail.com \\ Felipe Viegas Rodrigues \\ ORCID: https://orcid.org/0000-0002-1073-867X \\ Universidade do Oeste Paulista, Brasil \\ E-mail: rodrigues.fv@gmail.com
}

\begin{abstract}
Resumo
A doença de Chagas (DC) é transmitida pelo parasita hemoflagelado Trypanosoma cruzi e manifesta-se inicialmente de forma aguda e, depois, permanece na forma crônica, quando é caracterizada como indeterminada, cardíaca, digestiva ou mista. Alguns pacientes com doença de Chagas (DC) apresentam alterações cognitivas e há três hipóteses sobre os danos cognitivos: (I) alterações neurológicas e cognitivas causadas por consequência da forma cardíaca, (II) após a forma aguda há o desaparecimento espontâneo dos sinais clínicos da doença permanecendo as manifestações cognitivas, (III) as manifestações cognitivas e neurológicas são causadas por infecção crônica. O objetivo do presente trabalho foi fazer uma revisão sistemática da literatura para verificar os prejuízos cognitivos mensuráveis em pacientes chagásicos. Os artigos foram pesquisados nas bases Pubmed e Embase, com os descritores "Chagas disease" e "cognition". Foram incluídos artigos com mensuração de aspectos cognitivos de pacientes com a DC. Foram encontrados cento e noventa e sete artigos, três dos quais atendiam aos critérios. Todos os artigos demonstram prejuízos cognitivos relacionados à DC, forma crônica, com comprometimento tanto da inteligência geral quanto da memória de longa-duração, além de déficit no tempo de execução dos testes.
\end{abstract}

Palavras-chave: Cognição; Doença de Chagas; Função executiva; Revisão sistemática; Transtornos de memória.

\begin{abstract}
Chagas disease (CD) is transmitted by the hemoflagellate parasite Trypanosoma cruzi and manifests itself acutely and chronically, when it is characterized as indeterminate, cardiac, digestive or mixed. Some patients with Chagas' disease (CD) have cognitive alterations and there are three hypotheses about cognitive damage: (I) neurological and cognitive alterations caused by the cardiac form, (II) after the acute form there is the spontaneous disappearance of the clinical signs of disease with cognitive manifestations remaining, (III) cognitive and neurological manifestations are caused by chronic infection. The aim of the present study was to carry out a systematic review of the literature to verify measurable cognitive impairments in Chagas patients. The articles were searched in the Pubmed and Embase databases, with the descriptors "Chagas disease" and "cognition". All articles with assessment of cognitive aspects of patients with CD were included. One hundred and ninety-seven articles were found, three of which met the criteria. All articles demonstrate cognitive impairments related to $\mathrm{CD}$, in a chronic form, with impairment of both general intelligence and long-term memory, in addition to a deficit in the execution time of the tests.
\end{abstract}

Keywords: Cognition; Chagas disease; Executive function; Systematic review; Memory disorders.

\section{Resumen}

La enfermedad de Chagas (EC) es transmitida por el parásito hemoflagelado Trypanosoma cruzi y se manifiesta inicialmente de forma aguda y luego permanece en la forma crónica, cuando se caracteriza por ser indeterminada, cardíaca, digestiva o mixta. Algunos pacientes con enfermedad de Chagas (EC) presentan cambios cognitivos y existen tres hipótesis sobre el daño cognitivo: (I) cambios neurológicos y cognitivos provocados como consecuencia de la forma cardíaca, (II) después de la forma aguda hay una desaparición espontánea de la clínica. signos de enfermedad restantes manifestaciones cognitivas, (III) las manifestaciones cognitivas y neurológicas son causadas por una infección crónica. El objetivo de este estudio fue realizar una revisión sistemática de la literatura para verificar el deterioro cognitivo medible en pacientes chagásicos. Los artículos fueron buscados en las bases de datos Pubmed y Embase, con los descriptores "enfermedad de Chagas" y "cognición". Se incluyeron artículos que evaluaban aspectos cognitivos de 
pacientes con EC. Se encontraron ciento noventa y siete artículos, tres de los cuales cumplieron con los criterios. Todos los artículos demuestran un deterioro cognitivo relacionado con la EC, de forma crónica, con deterioro tanto de la inteligencia general como de la memoria a largo plazo, además de un déficit en el tiempo de ejecución de la prueba.

Palabras clave: Cognición; La enfermedad de Chagas; Función ejecutiva; Revisión sistemática; Trastornos de la memoria.

\section{Introdução}

A doença de Chagas (DC) ou a Tripanossomíase Americana é causada pelo parasito hemoflagelado Trypanosoma cruzi e pode ser transmitida de diversas formas. Entre os casos de doença de Chagas aguda confirmados no Brasil no período de 2000 a 2013, observou-se que a forma de transmissão oral foi a mais frequente (Ministério da Saúde, 2015). Segundo a Organização Pan-Americana de saúde (2017) na América Latina há aproximadamente seis milhões de pessoas infectadas, das quais quatorze mil vêm a óbito anualmente após contato com o protozoário.

A infecção pode ocorrer por transmissão vetorial, transfusão de sangue, transmissão congênita, transplante de órgãos, acidentes laboratoriais ou ainda pela forma oral. O parasito T. cruzi possui duas formas quando na infecção de mamíferos: a forma infectante tripomastigotas metacíclicas e as formas de replicação no hospedeiro vertebrado, chamadas formas amastigotas (Rassi Jr. et al. 2012). Uma vez transmitida, a doença se manifesta de forma aguda (DCA) por dois a quatro meses. Após esse período, ela é considerada crônica e pode durar mais de vinte anos em latência, denominada forma indeterminada (Villalta et al. 2013). Após o período de latência, muitos pacientes apresentam outras formas da doença, como a cardíaca (com prejuízos ao coração), a digestiva (que afeta cólon e esôfago), ou ainda a forma mista (que afeta tanto o coração quanto o cólon e esôfago).

Carlos Chagas $(1909,1911,1916)$, no entanto, também considerou a forma nervosa da doença, com danos ao sistema nervoso central e chegou a descrever lesões neuropatológicas em pacientes chagásicos, que seriam indicativos da presença do parasito no sistema nervoso central (SNC) de humanos. A forma nervosa foi descrita por Carlos Chagas, após autópsia em uma criança com DC que teve meningoencefalite e veio a óbito. Chagas (1909) observou desarmonias funcionais como condições intelectuais precárias, retardamento da evolução em crianças, ocorrência de convulsões com alta frequência em diversas idades, e infantilismo em adultos. Em diversas regiões do SNC encontraram lesões que foram denominadas de "Focos múltiplos disseminados", sendo que focos de inflamação foram encontrados desde o encéfalo até a medula espinhal, as múltiplas localizações foi o que atribuiu a expressão de variantes clínicas da síndrome nervosa. Além disso, perturbações motoras foram observadas em portadores da DC.

Artigos recentes corroboram os achados originais de Chagas (Prost et al., 2000; Walckermann et al, 2008; OliveiraFilho, 2009; Guimarães et al., 2014). Todos esses autores mostram uma significativa relação entre danos cognitivos e a doença de Chagas crônica, não somente na forma cardíaca da doença. No entanto, mais de cem anos após a descoberta da DC não se tem informações suficientes sobre as alterações comportamentais e as perdas cognitivas relacionadas à DC (Pereira, 2016).

Prost et al. (2000) argumentam que os pacientes crônicos da DC apresentam diferenças no traçado eletroencefalográfico (EEG) que são evidência de comprometimento encefálico nesta enfermidade, que não estaria relacionado à forma cardíaca da doença. Oliveira-Filho (2009), por outro lado, defende que a atrofia cerebral na DC crônica pode ter duas causas: a primeira sendo provocada pela cardiopatia chagásica que pode levar a um acidente vascular cerebral isquêmico (AVCi), que por sua vez causa a atrofia cerebral e perdas cognitivas e motoras. A segunda maneira é pela inflamação crônica que tem grande risco de levar diretamente a uma atrofia cerebral, ou provocar também um AVCi e, assim, à atrofia cerebral.

Além da forma nervosa da DC, a forma amastigota do T. cruzi no SNC vem ganhando espaço no debate de estudiosos pela elucidação de modificações comportamentais como distúrbios do sono, déficit de memória, perda da qualidade de vida e depressão, descrita em crianças e adultos jovens durante a fase crônica da DC (Jorg \& Rovira, 1981; Moncada, 1987; Silva et al. 2010). Os sintomas acima citados foram detectados em experimentos com Trypanosoma cruzi (Arankowsky-Sandoval et al. 
2001, Vilar-Pereira et al. 2012, 2015). Silva (2015) verificou que as citocinas IFN-y e TNF-alfa em ambiente inflamatório do SNC, levam a facilitação da introdução e o desenvolvimento do T. cruzi em astrócitos, sendo esta glia uma possível fonte do parasito em casos de reativação da infecção de pacientes com imunossupressão.

Portanto, o objetivo da presente revisão sistemática é investigar se pacientes chagásicos exibem prejuízos cognitivos mensuráveis, quais são eles e inferir se apoio psiquiátrico/psicológico também deve fazer parte da terapêutica destes indivíduos.

\section{Metodologia}

Trata-se de uma revisão sistemática de literatura de caráter observacional, retrospectivo e transversal, utilizando as recomendações PRISMA (Moher et al., 2009) para a organização dos resultados obtidos, sem restrição do ano de publicação.

\subsection{Indexadores}

A consulta foi feita aos bancos de dados indexadores Pubmed (US National Library of Medicine) e Embase (Elsevier ${ }^{\circledR}$ ), entre agosto e outubro de 2020, e considerados os seguintes descritores "Chagas disease" e "cognition".

\subsection{Critérios de elegibilidade}

Os critérios de inclusão adotados foram: todos os relatos de caso, estudos de coorte e estudos experimentais que tenham avaliado aspectos cognitivos dos pacientes chagásicos. As revisões de literatura foram excluídas, assim como os estudos experimentais em animais. $O$ processo de seleção dos artigos foi realizado por dois autores em conjunto. $\mathrm{O}$ critério de inclusão principal da revisão foi a realização de medida cognitiva em pacientes portadores da doença de Chagas. Artigos que apenas citavam o comprometimento cognitivo como possível dano causado pela doença de Chagas, e aqueles em que alguns pacientes da amostra total tinham queixas sobre danos cognitivos, mas sem testagem, foram excluídos.

Adotou-se a seguinte sequência no processo de amostragem: 1) inserção dos descritores nas bases de dados escolhidas; 2) construção de um arquivo (função exportar) com todos os artigos resultantes da busca; 3 ) leitura dos resumos, exclusão de estudos duplicados e observação da pertinência dos critérios de elegibilidade (busca por testes cognitivos em pacientes chagásicos); 4) aquisição da publicação completa nas bases de dados; 5) leitura completa dos artigos selecionados e exclusões adicionais (se necessário); 6) conclusão da amostra final.

\section{Resultados}

A partir dos descritores utilizados, foram encontrados cento e noventa e sete artigos, mas apenas três foram selecionados após seleção conforme os critérios de elegibilidade (Figura 1). 
Figura 1. Diagrama de fluxo das diferentes fases de seleção dos artigos incluídos na revisão sistemática.

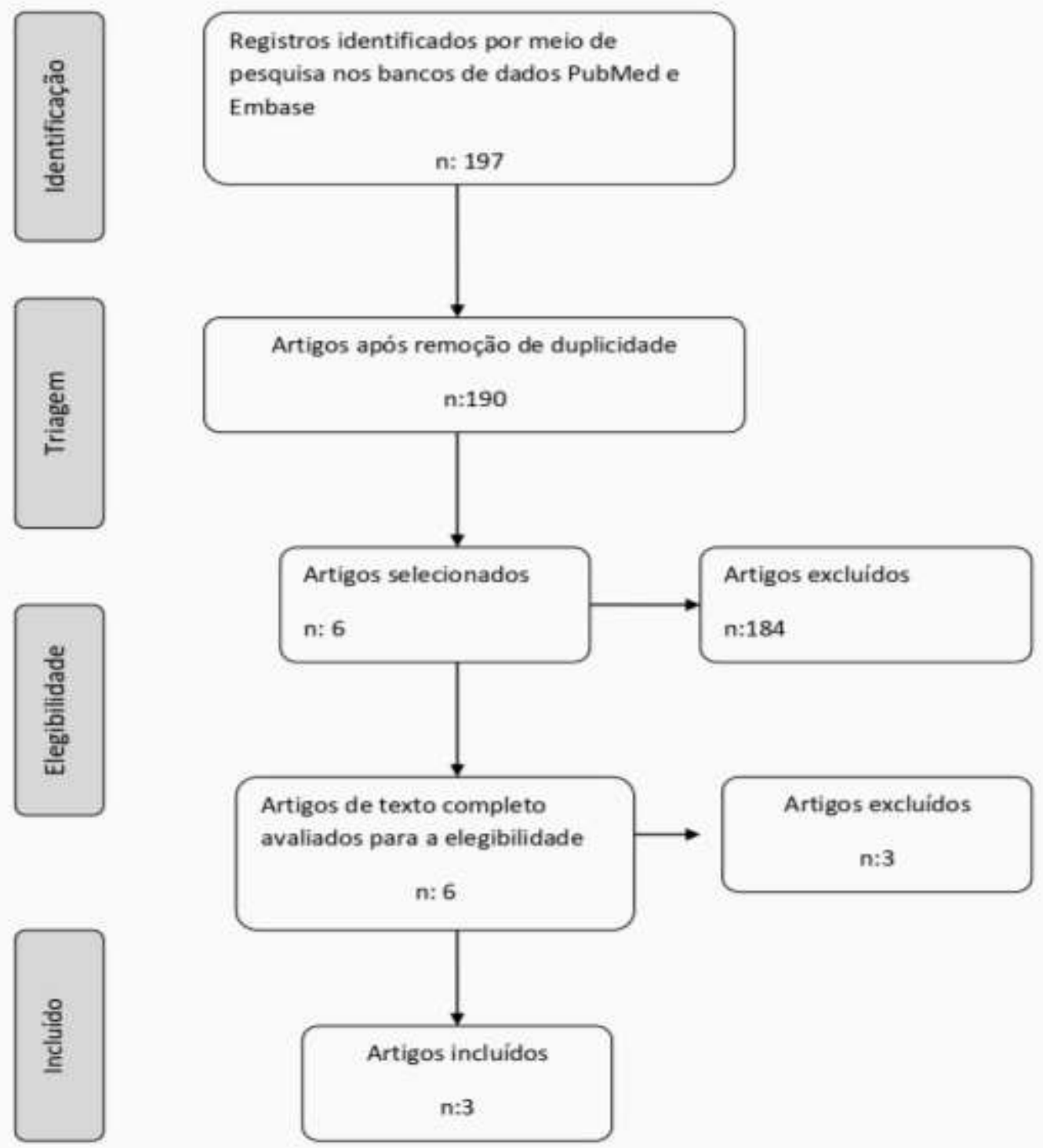

Fonte: Autores.

Os artigos resultantes da revisão, os únicos que efetivamente testaram funções cognitivas em pacientes com a DC, estão listados na Tabela 1 , incluindo os testes e funções avaliadas. 


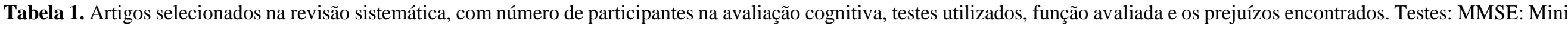

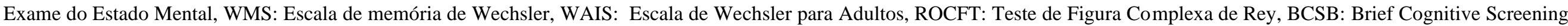
Battery.

\begin{tabular}{|c|c|c|c|c|}
\hline Autores & $\mathbf{N}^{0}$ participantes & Testes & Função avaliada & Prejuízo cognitivo \\
\hline $\begin{array}{c}\text { Mangone, Sica, Pereyra, Genovese, Segura, } \\
\text { Riarte \& Segura (1994) }\end{array}$ & $\begin{array}{l}45 \text { pacientes chagásicos } \\
26 \text { controles }\end{array}$ & $\begin{array}{l}\text { MMSE } \\
\text { WMS } \\
\text { WAIS }\end{array}$ & $\begin{array}{l}\text { Capacidade cognitiva geral e } \\
\text { específica, e memória. }\end{array}$ & $\begin{array}{c}\text { Atraso na resolução de } \\
\text { problemas }\end{array}$ \\
\hline $\begin{array}{c}\text { Dias, Lacerda, Vieira-de-Melo, Viana, Jesus, } \\
\text { Reis, Nitrini, Charcat-Fichman, Lopes \& } \\
\text { Oliveira-Filho (2009) }\end{array}$ & $\begin{array}{c}37 \text { pacientes com cardiomiopatia chagásica } \\
42 \text { pacientes com outras cardiomiopatias } \\
\text { (controles) }\end{array}$ & $\begin{array}{l}\text { MMSE } \\
\text { ROCFT } \\
\text { BCSB }\end{array}$ & $\begin{array}{l}\text { Capacidade cognitiva geral, } \\
\text { coeficiente de inteligência. }\end{array}$ & $\begin{array}{l}\text { Capacidade cognitiva } \\
\text { inferior ao controle }\end{array}$ \\
\hline $\begin{array}{l}\text { Lima-Costa, Castro-Costa, Uchôa, Firmo, } \\
\text { Ribeiro, Ferri \& Prince (2009) }\end{array}$ & $\begin{array}{c}1449 \text { habitantes, dos quais } 37,6 \% \text { eram } \\
\text { portadores de DC }\end{array}$ & MMSE & Capacidade cognitiva geral & $\begin{array}{l}\text { Capacidade cognitiva } \\
\text { inferior ao controle }\end{array}$ \\
\hline
\end{tabular}




\section{Discussão}

Todos os artigos selecionados apontam prejuízos cognitivos em pacientes com a DC (Mangone et al., 1994; Lima-Costa et al., 2009; Dias et al., 2009) e evidenciam comprometimento da inteligência geral e de funções específicas como memória de longa-duração. Mangone et. al. (1994) também relatam déficit no tempo de execução dos testes. Os artigos revelam que há associação entre as perdas da capacidade cognitiva com a doença de Chagas, principalmente nos artigos que utilizaram de métodos padronizados, que levaram em conta as características socioculturais da população alvo. Apenas Lima-Costa et al. (2009) também consideram que o dano cognitivo dos pacientes da DC pode ser decorrência da cardiomiopatia chagásica. Todos os demais são concordantes com uma forma nervosa da doença, como postulado originalmente por Chagas $(1909,1911)$.

Mangone et al. (1994) analisaram o desempenho cognitivo de 45 pacientes com a doença de Chagas crônica (DCC) que não tinham queixas sobre perdas cognitivas, através dos testes Mini-Exame do Estado Mental (MMSE), escala de memória de Wechsler (WMS), e escala de inteligência Wechsler para adultos (WAIS). Os autores concluem que existem perdas cognitivas em pacientes chagásicos, relacionado à velocidade de processamento, dado que eles apresentaram lentidão na resolução dos subtestes Dígitos e Procurar símbolos. Não houve diferença no desempenho do subteste Cubos, sugerindo que a percepção e o planejamento visuoespacial não estão afetados nos pacientes chagásicos.

Os autores formam a teoria de que as perdas cognitivas observadas podem ser causadas pela disfunção do lobo frontal, devido ao aumento do tempo necessário para a resolução dos problemas propostos. Este quadro é semelhante às disfunç̃oes cognitivas associadas à doença da substância branca descrita por Filley (2012), que relata a síndrome de WMD (white matter disease) proposta por Filley et al., (1988) e Franklin et al., (1989). Estes autores usaram a síndrome para descrever a demência que pode acompanhar o envolvimento da substância branca cerebral. Porém o tempo de reação central estudado com um paradigma excêntrico foi prejudicado em alguns desses pacientes.

Já Lima-Costa et al. (2009) utilizaram o teste MMSE para uma análise cognitiva funcional dos pacientes portadores da forma crônica da DC, além de realizarem um teste de eletrocardiograma (ECG) para verificar a relação entre perdas cognitivas (ou dano encefálico) com a cardiomiopatia chagásica. Os resultados mostram que a infecção por $T$. cruzi foi significativamente associada com menor pontuação no MMSE, sendo que a associação permanece mesmo depois dos ajustes para as anormalidades no ECG, uso atual de digoxina e para todas as outras variáveis de confusão potenciais.

Lima-Costa et al. (2009) não descartam, no entanto, que a medicação usada pelos pacientes para o tratamento das complicações cardíacas possa estar implicada com o dano cognitivo mensurado. Segundo o Cardiac Insufficiency Bisoprolol Study (CIBIS-I, 1999) na fase crônica o tratamento é sintomático para alívio das manifestações da insuficiência cardíaca. MaheutBosser (2006) descreve que a recomendação para esses pacientes são as medicações inibidores da enzima de conversão da angiotensina, betabloqueadores e digoxina. Dentre estes, apenas a digoxina foi associada previamente ao comprometimento da cognição. A dioxina não é mais considerada um tratamento medicamentoso de primeira linha (Aronow, 2005), porém 22\% dos pacientes da pesquisa que possuíam a doença de Chagas faziam uso da medicação. O efeito da medicação digoxina não diminuiu o efeito da DC sobre a pontuação MMSE, mesmo com o controle para a medicação o efeito da DC sobre o funcionamento cognitivo continuou grande e independente. Apesar dos argumentos colocados por Lima-Costa et al. (2009), não há, portanto, evidências que apoiem a hipótese que o uso do medicamento foi uma variável importante e interveniente.

Por fim, os autores ainda argumentam que a associação entre déficit cognitivo e a infecção crônica por T. cruzi pode ser explicada por doença cerebrovascular decorrente da cardiomiopatia chagásica. De fato, disfunção cognitiva vem sendo associada a insuficiência cardíaca, comumente acompanhada das síndromes clínicas que são associadas às doenças cerebrovasculares (Vogels et al, 2007; Selnes \& Vintes, 2006). O risco de AVC isquêmico é aumentado nas doenças cardíacas (Sacco, 1997). Em autópsias de pacientes chagásicos com insuficiência cardíaca foram encontrados infartos cerebrais (Aras et al, 2003), há evidências que o AVC isquêmico está associado à DCC (Carod-Artal et al, 2005). 
Vale notar que há relatos de alterações nervosas periféricas em pacientes chagásicos também, possivelmente associadas à infecção pelo T. cruzi. Wackerman et al. (2008) investigaram pacientes com DCC através do potencial evocado somatosensitivo e encontraram alterações importantes em sete dos vinte e dois pacientes investigados. Os autores defendem que os achados podem ser resultantes de uma neuropatia periférica em pacientes infectados pelo $T$. cruzi que não faziam uso de medicamentos para controle da infecção.

Dias et al. (2009) fazem uma comparação entre pacientes com cardiomiopatia chagásica (CD) e pacientes portadores de outras cardiomiopatias (OC), traçando paralelos entre as capacidades cognitivas deles. Os autores avaliaram setenta e nove pacientes, sendo trinta e sete destes com CD e quarenta e dois com OC. Os autores também utilizaram o MMSE, além do teste BCSB (do inglês Brief Cognitive Screening Battery) e, por fim, o teste da Figura Complexa de Rey (FCR). Os autores chegam a conclusão de que temos uma diferença entre os grupos, sendo a doença de Chagas associada a resultados anormais em testes cognitivos mesmo tendo como comparativos pacientes portadores de doenças cardíacas. Dias et al. (2009) também detectaram disfunção cognitiva em nove dos pacientes com DCC, e seis nos pacientes portadores de outras cardiomiopatias, mensurando que os pacientes chagásicos apresentam um desempenho pior na evocação tardia do FCR.

Corroborando com esses resultados temos Prost et al. (2000) que realizaram testes de eletroencefalograma (EEG) e potencial evocado P300 e detectaram diferenças eletrofisiológicas que servem como evidência para o comprometimento cerebral na DC, além disso, os autores também observaram um menor potencial da banda beta, e a existência de subgrupos com maior potencial de banda alfa. Wackermann et al. (2008), também detectaram três resultados anormais nas frequências tetas do EEG de pacientes chagásicos. Ambos os autores não encontraram relações entre o comprometimento cerebral e o miocárdico.

Nota-se que há uma escassez de artigos recentes na literatura sobre o estado cognitivo dos pacientes chagásicos, evidenciando que essa doença ainda é negligenciada. Os portadores da doença parecem não possuir apoio psicossocial para adequada integração à sociedade. Guimarães et al. (2014) mostraram correlação significante entre o suicídio e abuso de drogas lícitas e ilícitas entre os pacientes portadores do vírus HIV e com DC, ambas doenças crônicas.

Dentre os três artigos analisados todos evidenciam a teoria de que a doença de Chagas está associada a danos cognitivos, sendo que dois deles (Dias et al., 2009; Mangone et al., 1994) indicam que estes danos são independentes daqueles cardiovasculares, que impactam o sujeito do ponto de vista neurofuncional. Mesmo com essas evidências temos uma escassez de artigos sobre tais prejuízos: como se formam e como esse fator impacta na vida do sujeito do ponto de vista biopsicossocial. Os principais prejuízos parecem relacionar-se à velocidade de processamento e à memória.

\section{Considerações Finais}

Os prejuízos cognitivos causados aos pacientes chagásicos precisam ser devidamente remediados como parte do protocolo terapêutico utilizado, com o intuito oferecer melhores cuidados e mais atenção a pacientes com a DC crônica, resultando em melhor qualidade de vida. Há evidências para a defesa, de fato, de uma forma nervosa da doença. É urgente que avaliações mais completas sejam feitas nestes pacientes para poder elucidar tais perdas e assim melhor remediá-las, deixando de negligenciar a DC. Além desses fatores temos uma associação entre a doença de Chagas e o subdesenvolvimento e pobreza, tornando necessário estudos que informem a população sobre as práticas educativas em saúde, de forma a remediar o impacto social que a enfermidade causa aos seus portadores, melhorando a qualidade de vida.

\section{Referências}

Aras, R., Matta, J. A. M. D., Mota, G., Gomes, I., \& Melo, A. (2003). Cerebral infarction in autopsies of Chagasic patients with heart failure. Arquivos brasileiros de cardiologia, 81(4), 414-416. 
Aronow, W. S. (2005). Drug treatment of systolic and of diastolic heart failure in elderly persons. The Journals of Gerontology Series A: Biological Sciences and Medical Sciences, 60(12), 1597-1605.5.

Barreira, A. A., Said, G., \& Krettli, A. U. (1981). Multifocal demyelinative lesions of peripheral nerves in experimental chronic Chagas's disease. Transactions of the Royal Society of Tropical Medicine and Hygiene, 75(5), 751-751.

Carvalho, N. B. (2015). Manual de atendimento a pacientes com doença de Chagas: atenção básica 2014/2015. Faculdade de Medicina USP. São Paulo, 159218.

Cibis, I. I. (1999). Investigators and Committees. The cardiac insufficiency bisoprolol study II (CIBIS II). Lancet, $353(9), 13$.

Carod-Artal, F. J., Vargas, A. P., Horan, T. A., \& Nunes, L. G. N. (2005). Chagasic cardiomyopathy is independently associated with ischemic stroke in Chagas disease. Stroke, 36(5), 965-970.

Chagas, C. (1909). Nova tripanozomiaze humana: estudos sobre a morfolojia e o ciclo evolutivo do Schizotrypanum cruzi n. gen., n. sp., ajente etiolojico de nova entidade morbida do homem. Memórias do Instituto Oswaldo Cruz, 1(2), 159-218.

Chagas, C. (1911). Nova entidade morbida do homem: rezumo geral de estudos etiolojicos e clinicos. Memórias do Instituto Oswaldo Cruz, 3(2), 219-275.

Da Saude, M. (2010) Doença Aguda de Chagas. Revista de Patologia Tropical / Journal of Tropical Pathology, $36,4$.

De Faria C. R, Melo-Sousa S. E, \& Rassi A. (1982) Estudo neurofisiológico na doença de Chagas. Rev Goiana Med. 28(1/2):3-21.

DeFaria, C. R., Rezende, J. M. D., \& Rassi, A. (1988). Desnervação periférica nas diferentes formas clínicas da doença de Chagas. Arquivos de NeuroPsiquiatria, 46(3), 225-237.

Dias, J. S., Lacerda, A. M., Vieira-de-Melo, R. M., Viana, L. C., Jesus, P. A., Reis, F. J., Nitrini, R., Charcat-Fichman, H, Lopes A. A. \& Oliveira-Filho, J. (2009). Cognitive dysfunction in chronic Chagas disease cardiomyopathy. Dementia \& neuropsychologia, 3(1), 27-33.

Filley, C. M. (2012). White matter dementia. Therapeutic advances in neurological disorders, 5(5), 267-277.

Filley, C. M., Franklin, G. M., Heaton, R. K., \& Rosenberg, N. L. (1988). White matter dementia: clinical disorders and implications. Neuropsychiatry, Neuropsychology, \& Behavioral Neurology.

Franklin, G. M., Nelson, L. M., Filley, C. M., \& Heaton, R. K. (1989). Cognitive loss in multiple sclerosis: case reports and review of the literature. Archives of Neurology, 46(2), 162-167.

Jorg, M. E., \& Zalazar Rovira, I. (1981). Formas encefalopaticas de enfermedad de Chagas cronica observadas en Argentina. Memórias do Instituto Oswaldo Cruz, 76(4), 353-360.

Genovese, O. M., Sanz, O. P., Correale, J., Erro, M. G., \& Sica, R. E. P. (1989). Cerebral evoked potentials in human chronic Chagas' disease. Arquivos de neuro-psiquiatria, 47(3), 274-278.

Goin, J. C., Borda, E. S., Auger, S., Storino, R., \& Sterin-Borda, L. (1999). Cardiac M2 muscarinic cholinoceptor activation by human Chagasic autoantibodies: association with bradycardia. Heart, 82(3), 273-278.

Guimarães, P. M., Passos, S. R., Calvet, G. A., Hökerberg, Y. H., Lessa, J. L., \& Andrade, C. A. D. (2014). Suicide risk and alcohol and drug abuse in outpatients with HIV infection and Chagas disease. Brazilian Journal of Psychiatry, 36(2), 131-137.

Hagui, A., Souza, S.M., Lopes, D. S., Rodrigues, F.V. (2019) Assimetrias da atenção visuoespacial em idosos. Ciências \& Cognição, 24(1), 015-024.

Lai, M. K. P., Lai, O. F., Keene, J., Esiri, M. M., Francis, P. T., Hope, T., \& Chen, C. H. (2001). Psychosis of Alzheimer's disease is associated with elevated muscarinic M2 binding in the cortex. Neurology, 57(5), 805-811.

Lima-Costa, M. F., Castro-Costa, E., Uchôa, E., Firmo, J., Ribeiro, A. L. P., Ferri, C. P., \& Prince, M. (2009). A population-based study of the association between Trypanosoma cruzi infection and cognitive impairment in old age (the Bambuí Study). Neuroepidemiology, 32(2), 122-128.

Macedo, V., Prata, A., Silva, G. R., \& Castillo, E. (1982). Prevalência de alterações eletrocardiográficas em chagásicos (Informações preliminares sobre o Inquérito Eletrocardiográfico Nacional). Arq. bras. cardiol, 261-4.

Maheut-Bosser, A., Brembilla-Perrot, B., Hanesse, B., Piffer, I., \& Paille, F. (2006, October). Troubles cognitifs chez les sujets de plus de 65 ans induits par la prise de digoxine. In Annales de cardiologie et d'angeiologie (Vol. 55, No. 5, pp. 246-248). Elsevier Masson.

Mangone, C. A., Sica, R. E. P., Pereyra, S., Genovese, O., Segura, E., Riarte, A., ... \& Segura, M. (1994). Cognitive impairment in human chronic Chagas' disease. Arquivos de neuro-psiquiatria, 52(2), 200-203.

Oliveira-Filho, J. (2009). Stroke and brain atrophy in chronic Chagas disease patients: a new theory proposition. Dementia \& neuropsychologia, 3(1), 22-26.

Pan American Health Organization. (2017) Neglected infectious diseases. Chagas Disease https://www.paho.org/en/topics/Chagas-disease.

Pereira, G. V. (2016). Alterações neurológicas e comportamentais (ansiedade, depressão e memória) na fase crônica da infecção pelo Trypanosoma cruzi: modelo experimental e propostas terapêuticas (Doctoral dissertation).

Pitella, J. E. H. (1984). Ischemic cerebral changes in the chronic Chagasic cardiopathy. Arquivos de neuro-psiquiatria, $42(2), 105-115$. 
Research, Society and Development, v. 10, n. 9, e3710917549, 2021

(CC BY 4.0) | ISSN 2525-3409 | DOI: http://dx.doi.org/10.33448/rsd-v10i9.17549

Prost, Julio Oscar, Romero Villanueva, Horacio, Morikone, Ana María, Polo, Gustavo, \& Bosch, Ana María. (2000). Evidências de compromiso cerebral en el estadio crónico de la enfermedad de Chagas obtenidas por medio del potencial P300 y de electroencefalografía cuantificada. Arquivos de Neuro-Psiquiatria, $58(2 \mathrm{~A}), 262-271$.

Ribeiro, A. L. P., de Carvalho, A. C. C., Teixeira, M. M., Lombardi, F., \& Rocha, M. O. C. (2008). Chagas disease: impaired vagal modulation has been demonstrated, enhanced parasympathetic activity remains to be proved. International Journal of Cardiology, 123(3), 330-332.

Sacco, R. L. (1997). Risk factors, outcomes, and stroke subtypes for ischemic stroke. Neurology, 49(5 Suppl 4), S39-S44.

Said, G., Joskowicz, M., Barreira, A. A., \& Eisen, H. (1985). Neuropathy associated with experimental Chagas' disease. Annals of Neurology: Official Journal of the American Neurological Association and the Child Neurology Society, 18(6), 676-683.

Selnes, O. A., \& Vinters, H. V. (2006). Vascular cognitive impairment. Nature clinical practice neurology, 2(10), 538-547.

Silva, R. R. (2015) Efeitos de IFM03B3 e TNF sobre a infecção de astrócitos pelo Trypanosoma cruzi. Tese (Doutorado em Biologia Parasitária) - Fundação Oswaldo Cruz, Instituto Oswaldo Cruz, Rio de janeiro, RJ.

Villalta, F., Dobish, M. C., Nde, P. N., Kleshchenko, Y. Y., Hargrove, T. Y., Johnson, C. A., \& Lepesheva, G. I. (2013). VNI cures acute and chronic experimental Chagas disease. The Journal of infectious diseases, 208(3), 504-511.

Vogels, R. L., Scheltens, P., Schroeder-Tanka, J. M., \& Weinstein, H. C. (2007). Cognitive impairment in heart failure: a systematic review of the literature. European journal of heart failure, 9(5), 440-449.

Wackermann, P. V., Fernandes, R. M. F., Elias Jr, J., Dos Santos, A. C., Marques Jr, W., \& Barreira, A. A. (2008). Involvement of the central nervous system in the chronic form of Chagas' disease. Journal of the neurological sciences, 269(1-2), 152-157.

Zuchner, T., Schliebs, R., \& Perez-Polo, J. R. (2005). Down-regulation of muscarinic acetylcholine receptor M2 adversely affects the expression of Alzheimer's disease-relevant genes and proteins. Journal of neurochemistry, 95(1), 20-32. 